\title{
Some integrals involving $k$ gamma and $k$ digamma function
}

\author{
Saeed Ahmed
}

Correspondence:

saeedahmedshah54@gmail.com Govt Shah Hussain Degree College, Lahore, Pakistan

\section{Springer Open}

\begin{abstract}
In this paper, some new integrals involving $k$ gamma function and $k$ digamma function have been established. An integral is established involving $k$ gamma function, and its special values are discussed. Similarly, some new integrals have been established for $k$ digamma function, and different elementary function is associated with it for different values of $k$. A nice representation of the EulerMascheroni constant and $\pi$ in the form of $k$ digamma function for different values of $k$ is also obtained.

Keywords: $k$ gamma function, $k$ digamma function

Mathematics subject classification: 33B15, 41A58, 33C20
\end{abstract}

\section{$k$ gamma function}

The $k$ gamma function is a generalization of the classical gamma function introduced by Diaz and Pariguan [1], denoted and defined as

$$
\Gamma_{k}(z)=\lim _{n \rightarrow \infty} \frac{n ! k^{n}(n k)^{\frac{z}{k}-1}}{(z)_{n, k}}, k>0, z \in \mathbb{C} \backslash k \mathbb{Z}^{-} .
$$

The symbol for $(z)_{n, k}$ is called Pochhammer's $k$ symbol [2] and is defined as

$$
(z)_{n, k}=z(z+k)(z+2 k) \cdots(z+(n-1) k) .
$$

Due to (1.2), we see that (1.1) has simple poles at $0,-k,-2 k,-3 k, \cdots$. The residue of $k$ gamma function at these simple poles is $\frac{1}{(-1)^{n} k^{n} n !}$, see [3]. The integral form of $k$ gamma function is denoted and defined as [4]

$$
\Gamma_{k}(z)=\int_{0}^{\infty} e^{-\frac{t^{k}}{k}} t^{z-1} d t .
$$

The improper integral is convergent for $\operatorname{Re}(z)>0$. The $k$ gamma function reduces to the classical gamma function, i.e., $\Gamma_{k} \rightarrow \Gamma$ as $k \rightarrow 1$. A simple change of variable $t^{k}=k y$ reveals the relationship between $k$ gamma function and classical gamma function

(c) The Author(s). 2020 Open Access This article is licensed under a Creative Commons Attribution 4.0 International License, which permits use, sharing, adaptation, distribution and reproduction in any medium or format, as long as you give appropriate credit to the original author(s) and the source, provide a link to the Creative Commons licence, and indicate if changes were made. The images or other third party material in this article are included in the article's Creative Commons licence, unless indicated otherwise in a credit line to the material. If material is not included in the article's Creative Commons licence and your intended use is not permitted by statutory regulation or exceeds the permitted use, you will need to obtain permission directly from the copyright holder. To view a copy of this licence, visit http://creativecommons.org/licenses/by/4.0/. 


$$
\Gamma_{k}(z)=k^{\frac{z}{k}-1} \Gamma\left(\frac{z}{k}\right)
$$

The following properties of the $k$ gamma function have been discussed in $[5,6]$

$$
\begin{aligned}
& \Gamma_{k}(z+k)=z \Gamma_{k}(z), \\
& \Gamma_{k}(z) \Gamma_{k}(k-z)=\frac{\pi}{\sin \left(\frac{\pi z}{k}\right)} .
\end{aligned}
$$

In the "An integral involving the k gamma function" section, we will establish an integral involving $k$ gamma function and its special cases will also be discussed. In the "Stirling formula for the $k$ gamma function" section, the Stirling formula will be derived for the $k$ gamma function. In the "Some integrals representing digamma function" section, we will provide few integrals involving the $k$ digamma function. Few special cases of the $k$ digamma function will also be presented. In the "Euler-Mascheroni constant and $\mathrm{k}$ digamma function" section, we will find the relationship between the EulerMascheroni constant in the form $k$ digamma function for different values of $k$.

\section{An integral involving the $\boldsymbol{k}$ gamma function}

In this section, we will derive an interesting integral involving $k$ gamma function.

Theorem 2.1 Consider a complex number $p$ of the form $p=a+i b$. Then

$$
\begin{aligned}
& \frac{\Gamma_{k}(z)}{n|p|^{\frac{z}{k}} k^{\frac{z}{k}-1}} \cos \left(\frac{z \theta}{k}\right)=\int_{0}^{\infty} e^{-a u^{n}}\left(\cos b u^{n}\right) u^{\frac{n z}{k}-1} d u, \\
& \frac{\Gamma_{k}(z)}{n|p|^{\frac{z}{k}} k^{\frac{z}{k}-1}} \sin \left(\frac{z \theta}{k}\right)=\int_{0}^{\infty} e^{-a u^{n}}\left(\sin b u^{n}\right) u^{\frac{n z}{k}-1} d u .
\end{aligned}
$$

Proof Making the substitution $t=\left(k p u^{n}\right)^{\frac{1}{k}} \Rightarrow d t=p n u^{n-1}\left(k p u^{n}\right)^{\frac{1}{k}-1} d u$ into (1.3), we get

$$
\Gamma_{k}(z)=\int_{0}^{\infty} e^{-p u^{n}}\left(k p u^{n}\right)^{\frac{z-1}{k}} p n u^{n-1}\left(k p u^{n}\right)^{\frac{1}{k}-1} d u=n p^{\frac{z}{k}} k^{\frac{z}{k}-1} \int_{0}^{\infty} e^{-p u^{n}} u^{\frac{n z}{k}-1} d u
$$

so we get

$$
\frac{\Gamma_{k}(z)}{n p^{\frac{z}{k}} k^{\frac{z}{k}-1}}=\int_{0}^{\infty} e^{-p u^{n}} u^{\frac{n z}{k}-1} d u
$$

Similarly, for the conjugate of $p$, we can write

$$
\frac{\Gamma_{k}(z)}{n \bar{p}^{\frac{z}{k}} k^{\frac{z}{k}-1}}=\int_{0}^{\infty} e^{-\bar{p} u^{n}} u^{\frac{n z}{k}-1} d u
$$

Adding and simplifying (2.2) and (2.3), we get 


$$
\frac{\Gamma_{k}(z)}{n k^{\frac{z}{k}-1}}\left(\frac{1}{|p|^{\frac{z}{k}} e^{\frac{i z \theta}{k}}}+\frac{1}{|p|^{\frac{z}{k}} e^{-\frac{i \theta}{k}}}\right)=\int_{0}^{\infty}\left(e^{-(a+i b) u^{n}}+e^{-(a-i b) u^{n}}\right) u^{\frac{n z}{k}-1} d u .
$$

where $\theta$ and $|p|$ are the principal argument and modulus of $p$, respectively, so that (2.4) reduces to

$$
\frac{\Gamma_{k}(z)}{n|p|^{\frac{z}{k}} k^{\frac{z}{k}-1}}\left(\frac{1}{e^{\frac{i z \theta}{k}}}+\frac{1}{e^{-\frac{i \varepsilon \theta}{k}}}\right)=\int_{0}^{\infty} e^{-a u^{n}}\left(e^{-i b u^{n}}+e^{i b u^{n}}\right) u^{\frac{n z}{k}-1} d u .
$$

By Euler's identity, we can write

$$
\frac{\Gamma_{k}(z)}{n|p|^{\frac{z}{k}} k^{\frac{z}{k}-1}}\left(2 \cos \left(\frac{z \theta}{k}\right)\right)=\int_{0}^{\infty} e^{-a u^{n}}\left(2 \cos \left(b u^{n}\right)\right) u^{\frac{n z}{k}-1} d u .
$$

This yields the final integral (2.1a). Similarly, subtracting (2.2) and (2.3) and continuing in the same fashion, we get (2.1b).

Corollary 2.2 Take $a=0, b=1, n=1 \Rightarrow|p|=1, \theta=\frac{\pi}{2}$ in (2.1b), and using the relation (1.5) together with $k=1$, we see that

$$
\lim _{z \rightarrow 0}\left(\frac{\sin (z \pi / 2)}{z \pi / 2}\right) \lim _{z \rightarrow 0}\left(\frac{\pi z}{\sin (\pi z)}\right) \frac{\pi}{\Gamma(1)}\left(\frac{z \pi}{2 \pi z}\right)=\int_{0}^{\infty} \sin (u) u^{-1} d u
$$

This reduces to a well-known integral

$$
\int_{0}^{\infty} \frac{\sin u}{u} d u=\frac{\pi}{2}
$$

Corollary 2.3 Take $z=\frac{1}{2}, a=0, b=1, n=2 \Rightarrow p=1, \theta=\frac{\pi}{2}$ into (2.1b)

$$
\int_{0}^{\infty} \sin \left(u^{2}\right) u^{\frac{1}{k}-1} d u=\frac{\Gamma_{k}\left(\frac{1}{2}\right)}{2 k^{\frac{1}{2 k}}-1} \sin \left(\frac{\pi}{4 k}\right) .
$$

As $k \rightarrow 1$, the integral reduces to

$$
\int_{0}^{\infty} \sin \left(u^{2}\right) d u=\frac{\sqrt{2 \pi}}{4}
$$

Corollary 2.4 Take $z=\frac{1}{2}, k=1, b=0, n=2$ into (2.1a); it turns out the Gaussian integral

$$
\int_{0}^{\infty} e^{-a u^{2}} d u=\frac{1}{2} \sqrt{\frac{\pi}{a}}, a>0 .
$$

In general, for $m>0$ and $z=\frac{1}{m}, k=1, a=1, b=0$ into (2.1a), we get 


$$
\int_{0}^{\infty} e^{-u^{m}} d u=\frac{1}{m} \Gamma\left(\frac{1}{m}\right) .
$$

The integral (2.5) can also be written as

$$
\int_{-\infty}^{\infty} e^{-a u^{2}} d u=\sqrt{\frac{\pi}{a}}, a>0 .
$$

\section{Stirling formula for the $\boldsymbol{k}$ gamma function}

The Stirling formula is an approximation of the factorial for large $n$. It associates an appropriate function to the growth of $n$ ! which is given as

$$
\Gamma(n+1)=n ! \approx n^{n} e^{-n} \sqrt{2 \pi n}, n \in \mathbb{N} .
$$

In fact, it is quite accurate even for small $n$; for example, the Stirling formula gives $99 \%$ accuracy when compared with the value of 10! A formula similar to the Stirling formula can be obtained for the $k$ gamma function as follows:

Theorem 3.1 For $k>0, \operatorname{Re}(z)>0$,

$$
\Gamma_{k}(z+1)=\left(\frac{z}{e}\right)^{z / k} \sqrt{\frac{2 \pi}{k z^{1-2} / k}} .
$$

Proof Consider

$$
\Gamma_{k}(z+1)=\int_{0}^{\infty} e^{-\frac{t^{k}}{k}} t^{z} d t=\int_{0}^{\infty} e^{-\frac{t^{k}}{k}+z \ln t} d t .
$$

Now if we let $f(t)=-\frac{t^{k}}{k}+z \ln t$ and notice that its critical value is $f^{\prime}(t)=0 \Rightarrow t$ $=z^{1 / k}=a$ which gives maximum value $f^{\prime}(a)=-k z^{1-2} / k<0$ for $k>0$. Now if we expand the function $f(t)$ by Taylor series around its critical point, we get

$$
f(t)=f(a)+(t-a) f^{\prime}(a)+\frac{(t-a)^{2}}{2 !} f^{\prime^{\prime}}(a)+O\left((t-a)^{3}\right) .
$$

Since $a$ is the critical point of the function, the second term of the series vanishes, and the rest simplifies to

$$
f(t)=-\frac{z}{k}+\frac{z}{k} \ln (z)-\frac{k}{2} z^{1-2 / k}\left(t-z^{1 / k}\right)^{2}+O\left(\left(t-z^{1 / k}\right)^{3}\right) .
$$

Substituting it into (3.3) and ignoring the higher order terms, we get

$$
\Gamma_{k}(z+1)=z^{z / k} e^{-\frac{z}{k}} \int_{0}^{\infty} e^{-\frac{k}{2} z^{1-2} / k\left(t-z^{1 / k}\right)^{2}} d t .
$$

Substituting

$$
t-z^{1 / k}=y
$$




$$
\Gamma_{k}(z+1)=z^{z / k} e^{-\frac{z}{k}} \int_{-z 1 / k}^{\infty} e^{-\frac{k}{2} z^{1-2 / k}\left(t-z^{1 / k}\right)^{2}} d t .
$$

Since the integrand of the integral in (3.4) is a Gaussian curve whose peak, the maximum value, lies at $t=z^{1 / k}$ so at $t<0$ the integral is negligible. Therefore, we can extend the lower limit to $-\infty$

$$
\Gamma_{k}(z+1) \approx z^{z} / k e^{-\frac{z}{k}} \int_{-\infty}^{\infty} e^{-\frac{k}{2} z^{1-2} / k(t-z / k)^{2}} d t .
$$

Using (2.6), we can write as

$$
\Gamma_{k}(z+1)=z^{z / k e^{-\frac{z}{k}}} \sqrt{\frac{\pi}{\frac{k}{2} z^{1-2} / k}} .
$$

This simplifies to (3.2) as claimed. Notice that for $k \rightarrow 1$, (3.5) reduces to (3.1).

\section{Some integrals representing digamma function}

The logarithmic derivative of the $k$ gamma function for $\operatorname{Re}(z), k>0$ is known as $k$ digamma function, denoted and defined as [7]

$$
\psi_{k}(z)=\frac{\partial}{\partial z} \log \Gamma_{k}(z)=\frac{\Gamma_{k}^{\prime}(z)}{\Gamma_{k}(z)} .
$$

Taking the logarithmic derivative of the relation (1.4), we see that

$$
\frac{\partial}{\partial z} \log \Gamma_{k}(z+k)=\frac{\partial}{\partial z} \log z+\frac{\partial}{\partial z} \log \Gamma_{k}(z)
$$

Using (4.1), we can write

$$
\psi_{k}(z+k)=\psi_{k}(z)+\frac{1}{z} .
$$

The relation (4.2) is sometimes called the functional equation of $k$ digamma function.

Remark 4.1 Notice that for $k \rightarrow 1, \psi_{k}(z) \rightarrow \psi(z)$.

A series representation of $k$ digamma function is derived in [3] by taking the logarithmic derivative of the inverse of $k$-analogue Weierstrass form of the $k$ gamma function

$$
\Gamma_{k}(z)=z^{-1} k^{\frac{z}{k}} e^{-\frac{z}{k} \gamma} \prod_{n=1}^{\infty}\left(\frac{n k}{z+n k}\right) e^{\frac{z}{n k}}
$$

And is given by

$$
\psi_{k}(z)=-\frac{1}{z}+\frac{1}{k} \log k-\frac{\gamma}{k}+\sum_{n=1}^{\infty}\left(\frac{1}{n k}-\frac{1}{z+n k}\right) .
$$

where $\gamma$ is the Euler-Mascheroni constant given by the following series form 


$$
\gamma=\sum_{n=1}^{\infty} \frac{1}{n}-\log (n)=0.5772156649
$$

We can rearrange the series (4.3) to write

$$
\psi_{k}(z)=\frac{1}{k} \log k-\frac{\gamma}{k}+\sum_{n=0}^{\infty}\left(\frac{1}{(n+1) k}-\frac{1}{n k+z}\right) .
$$

Next, we derive some integrals involving $k$ digamma function.

Theorem 4.2 Let $s>0$ be a real number then for $k>0$

$$
\begin{aligned}
& \int_{0}^{1} \frac{x^{s-1}}{1+x^{k}} d x=\frac{1}{2}\left[\psi_{k}\left(\frac{s+k}{2}\right)-\psi_{k}\left(\frac{s}{2}\right)\right]=\psi_{k}(s)-\psi_{k}\left(\frac{s}{2}\right)-\frac{1}{k} \log (2), \\
& \int_{0}^{1} \frac{x^{s-1}\left(1-x^{(n+1) k}\right)}{1-x^{2 k}} d x=\frac{1}{2}\left(\psi_{k}\left(\frac{s+(n+1) k}{2}\right)-\psi_{k}\left(\frac{s}{2}\right)\right), \\
& \int_{0}^{1} \frac{1}{x\left(1+x^{k}\right)} \sum_{n=0}^{\infty} x^{\frac{s}{2^{n}}} d x=\psi_{k}(s)-\psi_{k}\left(\frac{s}{2^{n+1}}\right)-\frac{n+1}{k} \log (2), \\
& \int_{0}^{\infty} \tanh (k x) e^{-s x} d x=\frac{1}{2}\left[\psi_{k}\left(\frac{s+2 k}{4}\right)-\psi_{k}\left(\frac{s}{4}\right)-\frac{2}{s}\right] .
\end{aligned}
$$

Proof Using the Taylor series of $\frac{1}{1+x^{k}}$ the LHS of (4.5) becomes

$$
\int_{0}^{1} \frac{x^{s-1}}{1+x^{k}} d x=\int_{0}^{1} \sum_{n=1}^{\infty}(-1)^{n-1} x^{k n-k+s-1} d x
$$

Interchanging integral and summation

$$
=\sum_{n=1}^{\infty}(-1)^{n-1} \int_{0}^{1} x^{k n-k+s-1} d x=\sum_{n=1}^{\infty}(-1)^{n-1}\left(\frac{1}{k n-k+s}\right) .
$$

Rearranging the sum into even and odd terms, we can write

$$
=\sum_{n=1}^{\infty}\left(\frac{1}{2 n k-2 k+s}-\frac{1}{2 n k-k+s}\right) .
$$

Adding and subtracting $\frac{1}{2 n k}$ under the summation and factoring out $\frac{1}{2}$, we get

$$
=\frac{1}{2} \sum_{n=1}^{\infty}\left(\frac{1}{n k}-\frac{1}{n k+\frac{s-k}{2}}\right)-\frac{1}{2} \sum_{n=1}^{\infty}\left(\frac{1}{n k}-\frac{1}{n k+\frac{s-2 k}{2}}\right) .
$$

Changing the index of the sum, we get 


$$
=\frac{1}{2} \sum_{n=0}^{\infty}\left(\frac{1}{(n+1) k}-\frac{1}{n k+\frac{s+k}{2}}\right)-\frac{1}{2} \sum_{n=0}^{\infty}\left(\frac{1}{(n+1) k}-\frac{1}{n k+\frac{s}{2}}\right) .
$$

Adding and subtracting $(\log k) / k-\gamma / k$ and using (4.4), we get the first equality of (4.5). To prove the second equality of (4.5), we take Legendre duplication $k$ analogous formula $r=2$ in corollary 3.14 in [8]

$$
\Gamma_{k}(2 z)=2^{\frac{2 z}{k}-\frac{1}{2}} k^{\frac{1}{2}}(2 \pi)^{-\frac{1}{2}} \Gamma_{k}(z) \Gamma_{k}\left(z+\frac{k}{2}\right) .
$$

Taking the logarithm of (4.8)

$$
\begin{aligned}
\log \Gamma_{k}(2 z)= & \left(\frac{2 z}{k}-\frac{1}{2}\right) \log 2+\frac{1}{2} \log k-\frac{1}{2} \log (2 \pi)+\log \Gamma_{k}(z) \\
& +\log \Gamma_{k}\left(z+\frac{k}{2}\right) .
\end{aligned}
$$

Taking derivatives of (4.9) with respect to $z$ and using the definition (4.1), we get

$$
\psi_{k}(2 z)=\frac{1}{2}\left\{\psi_{k}(z)+\psi_{k}\left(z+\frac{k}{2}\right)\right\}+\frac{1}{k} \log (2) .
$$

Replacing $z$ by $s / 2$ in (4.10)

$$
\psi_{k}(s)=\frac{1}{2}\left\{\psi_{k}\left(\frac{s}{2}\right)+\psi_{k}\left(\frac{s+k}{2}\right)\right\}+\frac{1}{k} \log (2) .
$$

Rearranging we get the second equality of (4.5).

$$
\frac{1}{2}\left\{\psi_{k}\left(\frac{s+k}{2}\right)-\psi_{k}\left(\frac{s}{2}\right)\right\}=\psi_{k}(s)-\psi_{k}\left(\frac{s}{2}\right)-\frac{1}{k} \log (2) .
$$

To derive (4.6a), we replace $s$ by $s+k, s+2 k, s+3 k, \cdots s+n k$ in (4.5); we get

$$
\begin{gathered}
\int_{0}^{1} \frac{x^{s+k-1}}{1+x^{k}} d x=\frac{1}{2}\left(\psi_{k}\left(\frac{s+2 k}{2}\right)-\psi_{k}\left(\frac{s+k}{2}\right)\right) \\
\int_{0}^{1} \frac{x^{s+2 k-1}}{1+x^{k}} d x=\frac{1}{2}\left(\psi_{k}\left(\frac{s+3 k}{2}\right)-\psi_{k}\left(\frac{s+2 k}{2}\right)\right) \\
\int_{0}^{1} \frac{x^{s+n k-1}}{1+x^{k}} d x=\frac{1}{2}\left(\psi_{k}\left(\frac{s+(n+1) k}{2}\right)-\psi_{k}\left(\frac{s+n k}{2}\right)\right) .
\end{gathered}
$$

Now observing that the RHS of (4.11) is a telescoping sum, so adding all the $(n+1)$ terms in (4.11), we get

$$
\int_{0}^{1} \frac{x^{s-1}\left(1+x^{k}+x^{2 k}+\cdots x^{n k}\right)}{1+x^{k}} d x=\frac{1}{2}\left(\psi_{k}\left(\frac{s+(n+1) k}{2}\right)-\psi_{k}\left(\frac{s}{2}\right)\right) .
$$

The series under the integral on LHS of (4.12) is a finite geometric series with common ratio $x^{k}$, so summing it, we get (4.6a). In a similar fashion, we can derive (4.6b) by successively replacing $s$ by $s / 2, s / 2^{2}, s / 2^{3}, \cdots s / 2^{n}$ in the second equality of (4.5). 
Remark 4.3 The ratio test shows that the infinite series $\sum_{n=0}^{\infty} x^{\frac{s}{2^{n}}}$ is convergent for all real value of $x$ as long as $s>0$.

For the relation (4.7), first, we write the integrand as an exponential function

$$
\int_{0}^{\infty} \tanh (k x) e^{-s x} d x=\int_{0}^{\infty} \frac{1-e^{-2 k x}}{1+e^{-2 k x}} e^{-s x} d x
$$

By making the substitution $k x=-\log \sqrt{t^{k}} \Rightarrow d x=-\frac{1}{2 t} d t$ in (4.13), we can write

$$
\int_{0}^{\infty} \tanh (k x) e^{-s x} d x=\frac{1}{2} \int_{0}^{1} \frac{1-t^{k}}{1+t^{k}} t^{\frac{s}{2}-1} d t=\frac{1}{2} \int_{0}^{1} \frac{t^{\frac{s}{2}-1}}{1+t^{k}} d t-\frac{1}{2} \int_{0}^{1} \frac{t^{\frac{s}{2}+k-1}}{1+t^{k}} d t .
$$

By using the first equality of (4.5), we can write

$$
\int_{0}^{\infty} \tanh (k x) e^{-s x} d x=\frac{1}{2}\left[\frac{1}{2}\left(\psi_{k}\left(\frac{s+2 k}{4}\right)-\psi_{k}\left(\frac{s}{4}\right)\right)-\frac{1}{2}\left(\psi_{k}\left(\frac{s+4 k}{4}\right)-\psi_{k}\left(\frac{s+2 k}{4}\right)\right)\right] .
$$

After a bit simplification

$$
\int_{0}^{\infty} \tanh (k x) e^{-s x} d x=\frac{1}{2}\left[\psi_{k}\left(\frac{s+2 k}{4}\right)-\frac{1}{2} \psi_{k}\left(\frac{s}{4}\right)-\frac{1}{2} \psi_{k}\left(k+\frac{s}{4}\right)\right] .
$$

Using the relation (4.2), Eq. (4.14) reduces to the required result (4.7)

Remark 4.4 Equation (4.7) can be also be written in the form of Laplace transform of $\tanh (k x)$, that is

$$
L(\tanh (k x))=\frac{1}{2}\left[\psi_{k}\left(\frac{s+2 k}{4}\right)-\psi_{k}\left(\frac{s}{4}\right)-\frac{2}{s}\right] .
$$

Special values: Replacing $k=1=s$ in the first equality of (4.5), we can write

$$
\log (2)=\frac{1}{2}\left[\psi(1)-\psi\left(\frac{1}{2}\right)\right] .
$$

Replacing $k=2, s=1$ and for $k=4, s=2$, we get from (4.5)

$$
\frac{\pi}{2}=\psi_{2}\left(\frac{3}{2}\right)-\psi_{2}\left(\frac{1}{2}\right)=2\left(\psi_{4}(3)-\psi_{4}(1)\right) .
$$

Replacing $k=1 / 2, s=1$, we get from (4.5)

$$
\int_{0}^{1} \frac{1}{1+\sqrt{x}} d x=2-2 \log (2)=\frac{1}{2}\left[\psi_{\frac{1}{2}}\left(\frac{3}{4}\right)-\psi_{\frac{1}{2}}\left(\frac{1}{2}\right)\right] \text {. }
$$

\section{Euler-Mascheroni constant and $\boldsymbol{k}$ digamma function}

In this section, we represent the Euler-Mascheroni constant in the form $k$ digamma function for different values of $k$.

Proposition 5.1 Substituting $k=4$ and $z=1$ in (4.3), we see that 


$$
\psi_{4}(1)=-1+\frac{1}{4} \log 4-\frac{\gamma}{4}+\sum_{n=1}^{\infty}\left(\frac{1}{4 n}-\frac{1}{4 n+1}\right)
$$

The series in (5.1) is not hard to sum. First, observe that this series can be written as

$$
\begin{aligned}
\sum_{n=1}^{\infty}\left(\frac{1}{4 n}-\frac{1}{4 n+1}\right) & =\sum_{n=1}^{\infty} \int_{0}^{1}\left(x^{4 n-1}-x^{4 n}\right) d x=\int_{0}^{1} \sum_{n=1}^{\infty}\left(x^{4 n-1}-x^{4 n}\right) d x \\
& =\int_{0}^{1}\left(x^{3} \sum_{n=1}^{\infty}\left(x^{4}\right)^{n-1}-x^{4} \sum_{n=1}^{\infty}\left(x^{4}\right)^{n-1}\right) d x
\end{aligned}
$$

Now employing the Taylor series

$$
\begin{aligned}
=\int_{0}^{1}\left(\frac{x^{3}}{1-x^{4}}-\frac{x^{4}}{1-x^{4}}\right) d x & =\int_{0}^{1} \frac{x^{3}}{(1+x)\left(1+x^{2}\right)} d x \\
& =\int_{0}^{1}\left(1-\frac{1}{2(1+x)}-\frac{x+1}{2\left(1+x^{2}\right)}\right) d x .
\end{aligned}
$$

Evaluating the integral, we get the sum of the series

$$
\sum_{n=1}^{\infty}\left(\frac{1}{4 n}-\frac{1}{4 n+1}\right)=1-\frac{\pi}{4}-\frac{3}{4} \log (2) .
$$

Substituting (5.2) into (5.1), we get a nice representation of the Euler-Mascheroni constant in the form of 4-digamma function

$$
\gamma=-4 \psi_{4}(1)-\frac{\pi}{2}-\log (2)
$$

Or more elegantly

$$
\pi+2 \gamma=-8 \psi_{4}(1)-2 \log (2) .
$$

From (4.15) and (5.3), we get some more such representations

$$
\begin{gathered}
\gamma=-4 \psi_{4}(3)+\frac{\pi}{2}-\log (2), \\
\gamma=-4 \psi_{4}(3)+\psi_{2}\left(\frac{3}{2}\right)-\psi_{2}\left(\frac{1}{2}\right)-\log (2), \\
\gamma=-4 \psi_{4}(3)+\psi_{2}\left(\frac{3}{2}\right)-\psi_{2}\left(\frac{1}{2}\right)+\frac{1}{4} \psi_{\frac{1}{2}}\left(\frac{3}{4}\right)-\frac{1}{4} \psi_{\frac{1}{2}}\left(\frac{1}{2}\right)-1 .
\end{gathered}
$$

Adding (5.3) and (5.4), we get such representation for $\pi$

$$
\pi=4\left[\psi_{4}(3)-\psi_{4}(1)\right] \text {. }
$$

\section{Conclusion}

In this paper, we established and investigated few new definite integrals involving $k$ gamma function and $k$ digamma function. Known results of the classical gamma function and classical digamma function were obtained as special cases of $k$ gamma function and $k$ digamma function. We also established nice representations of $\pi$ and of the Euler-Mascheroni constant which were generated, and many more can still be obtained. 
Acknowledgements

Not applicable.

\section{Author's contributions}

Ahmed S. contributed the whole research article and approved the final manuscript.

Funding

There are no funding sources for this manuscript.

Availability of data and materials

Data sharing is not applicable to this article as no datasets were generated or analyzed during the current study.

\section{Competing interests}

The author declares that there are no competing interests.

Received: 31 March 2020 Accepted: 8 July 2020

Published online: 25 July 2020

\section{References}

1. Diaz, R., Pariguan, E.: On hypergeometric functions and k-Pochhammer symbol. Divulg. Mat. 15(2), 179-192 (2007)

2. Kokologiannaki, C.G.: Properties and inequalities of generalized k-gamma, beta and zeta functions. Int J Contemp Math Sci. 5(14), 653-660 (2010)

3. Mubeen, S., Rehman, A., Shaheen, F.: Properties of k-gamma, k-beta and k-psi functions. Bothalia. 44(4), 372-380 (2014)

4. Kokologiannaki C.G, Krasniqi V: Some properties of k-gamma function, Le Matematiche, 2013, LXVIII: 13 - 22.

5. Mansoor, M.: Determining the k-generalized gamma function Gamma_k(x), by functional equations. Int J Contemp Math Sci. 4(21), 1037-1042 (2009)

6. Mubeen, S., Habibullah, G.M.: An integral representation of some k-hypergeometric functions. Int Math Forum. 7(4), 203207 (2012)

7. Krasniqi, V:: A limit for the k-gamma and k-beta function. Int Math Forum. 5(33), 1613-1617 (2010)

8. Mubeen, S., Rehman, A.: A note on k-Gamma function and Pochhammer k-symbol. J Inform Math Sci. 6(2), 93-107 (2014)

\section{Publisher's Note}

Springer Nature remains neutral with regard to jurisdictional claims in published maps and institutional affiliations.

\section{Submit your manuscript to a SpringerOpen ${ }^{\circ}$ journal and benefit from:}

- Convenient online submission

Rigorous peer review

- Open access: articles freely available online

High visibility within the field

- Retaining the copyright to your article 